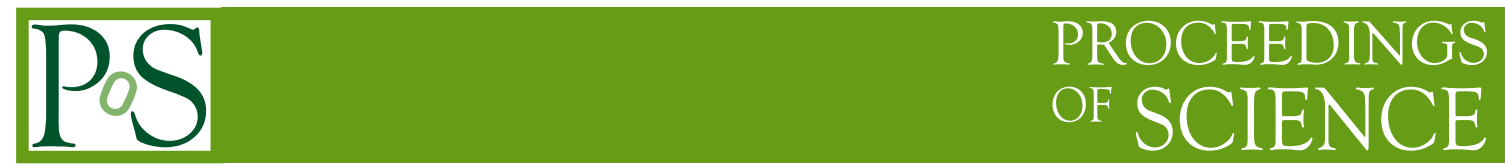

\title{
Upgrade of the CMS Pixel Detector
}

\section{S. König*}

Paul Scherrer Institut, Villigen PSI 5232, Switzerland

E-mail: stefan.koenig@psi.ch

The pixel detector of the CMS experiment will suffer from radiation damage. The innermost detectors are designed for two years of full LHC luminosity or an integrate luminosity of about $1 \mathrm{fb}^{-1}$. Afterwards they need to be replaced. On the other hand, plans for a step wise luminosity upgrade of the accelerator beyond the present design value are being prepared. The replacement pixel detector must be designed to handle increased particle rates and it should maintain or improve the tracking performance of the present system while being compatible with existing services.

VERTEX 2009 - $18^{\text {th }}$ International Workshop on Vertex detectors

September 13-18, 2009,

VELUWE, the Netherlands

${ }^{*}$ Speaker. 


\section{Upgrade Plans}

The innermost tracking detector of the CMS experiment located at the LHC collider ring at CERN is the pixel detector [1]. It is composed of 3 barrel layers and two endcap disks at each end. A sketch of the CMS pixel system is shown in figure 1.

In the process of increasing the luminosity of the LHC collider, a peak instantaneous luminosity of $2 \times 10^{34} \mathrm{~cm}^{-2} \mathrm{~s}^{-1}$ is expected by the year 2018 [2]. By this time the innermost layers of the pixel system have already taken a considerable amount of radiation damage. They cannot withstand the increased radiation levels after the upgrade. Furthermore, the readout inefficiencies at those luminosities will be unacceptably high and demand a change in the readout chip and data links. Therefore, a complete exchange of the pixel system is planned [3].

The high particle rates and intense radiation environment of the upgrade scenario demands a pixel detector with improved performance with respect to the existing one. This task is even more complicated as the existing infrastructure of cooling lines, power cables and readout fibers cannot be changed for the upgraded system. Also the mechanical envelope of the system has to be the same as the current one.

\subsection{Upgraded Pixel Detector}

It is planned to insert a system better suited for the higher occupancy environment and at the same time having a lower material budged than the current pixel detector. In order to achieve the first goal, modifications in the whole readout chain are needed. This includes the readout chip as well as the module controller and the development of a new flex hybrid.

The reduction of the material budget will be achieved by a bundle of measures. The introduction of a $\mathrm{CO}_{2}$ based cooling system, a consequently lightweight design of the support structure and modified pixel modules will substantially reduce the overall material effects compared to the current pixel system. In addition material from the detector end flange region and the supply tube will be

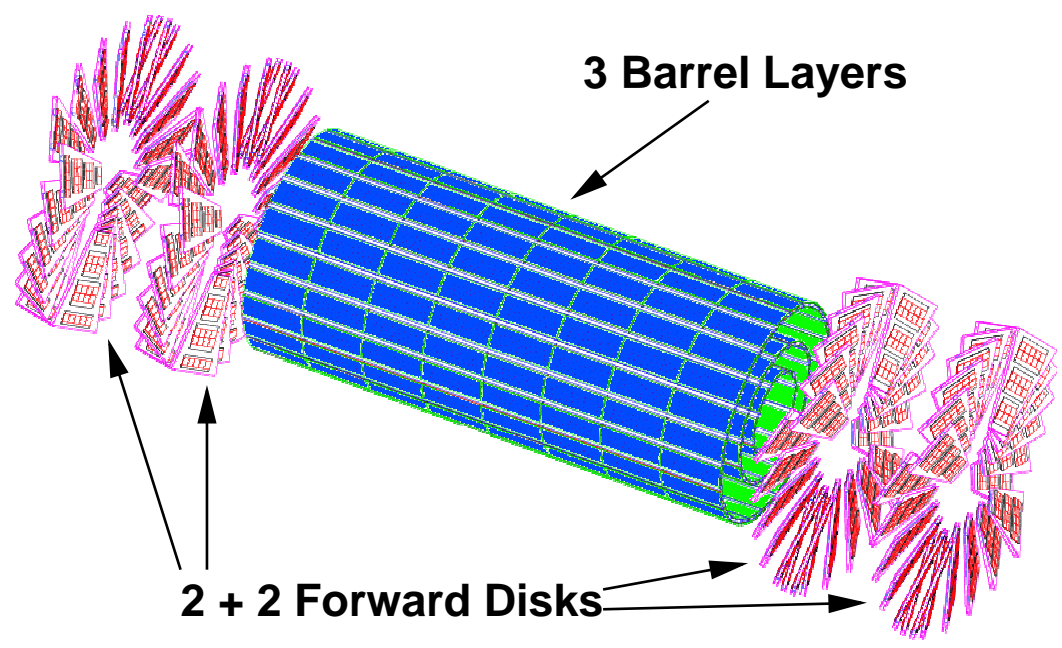

Figure 1: Drawing of the CMS pixel detector. Three barrel layers are accompanied by two endcap disks on each side allow three pixel hits per track in a region up to eta $=2.5$. 
shifted out of the sensitive tracking region. This is possible due to the rearrangement of optical conversion and power distribution boards towards higher pseudorapidities $|\eta|>2$. Alltogether the reduction in material budged will be such that an additional detector layer can be introduced and still keeping the material effects of the upgraded pixel lower than the existing detector. With the additional layer and the third disks the upgraded system is capable of measuring 4 space points up to $|\eta|=2.5$ with a small area of three hit coverage in the barrel to endcap transition region at $|\eta| \approx 1.3$. This not only enhances the robustness of the track finding and allows for a limited standalone tracking of the pixel detector, but also reduces the effect of unavoidable inefficiencies of the system due to electronics inefficiencies and non-hermetical coverage of layers with sensors. The increased number of modules (from today 768 to new 1216 in the barrel) together with the higher particle rate leads to an increased power demand of the new system. In order to be able to use the existing cables and power supplies, a DC to DC converter stage will be introduced to reduce the currents and the power losses in the cables.

In the following sections the two building blocks of the detector are described in more detail. Afterwards the module design is introduced. In the last two sections the module readout and powering issues are addressed.

\section{Mechanics}

The design principle for the mechanics of the upgraded pixel system is similar to the existing one (see fig. 2). The detector is composed of two half barrels accompanied by two couples of half disks on each side. Each part has its own supply half cylinder, called supply tube, that carries the services in form of cooling pipes, power lines, optical fibers and other cables. While the forward disks are integrated in their supply cylinders, the barrel part of the pixel detector is connected to the supply tubes by a flexible interface. This allows for a certain amount of bending between the individual parts during insertion.

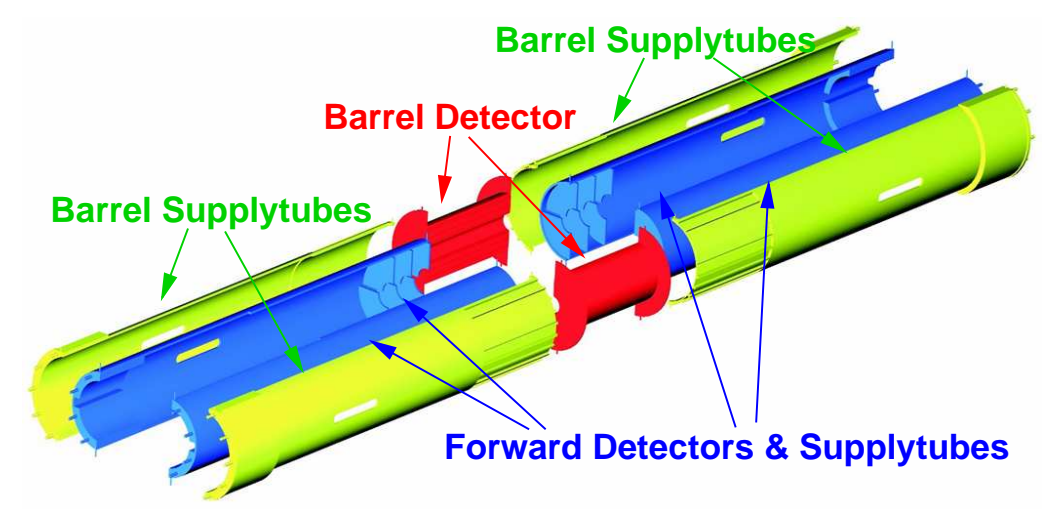

Figure 2: Drawing showing the CMS pixel detector with the service cylinders called supply tubes. The outermost supply tube holds the services for the barrel pixel detector. The forward pixel detector is incorporated in the innermost supply tubes. 


\subsection{Barrel Mechanics}

The barrel is divided into two half shells which are composed of three individual half barrel layers (see fig. 3). The new barrel part consists of 4 instrumented layers with mean radii of 39, 68, 109 and $160 \mathrm{~mm}$. The modules are arranged on 16 to 64 facets in phi and 8 modules along the beam pipe (see fig. 4). In total 1216 modules will be needed to populate the barrel system. All services are carried by a supply tube at each end of the barrel detector, which are connected to the detector via carbon fiber hinges at each end of the barrel. This somewhat flexible fixation allows for small bendings ( $\approx 3$ degrees) which occur during the insertion of the detector system into the CMS experiment.

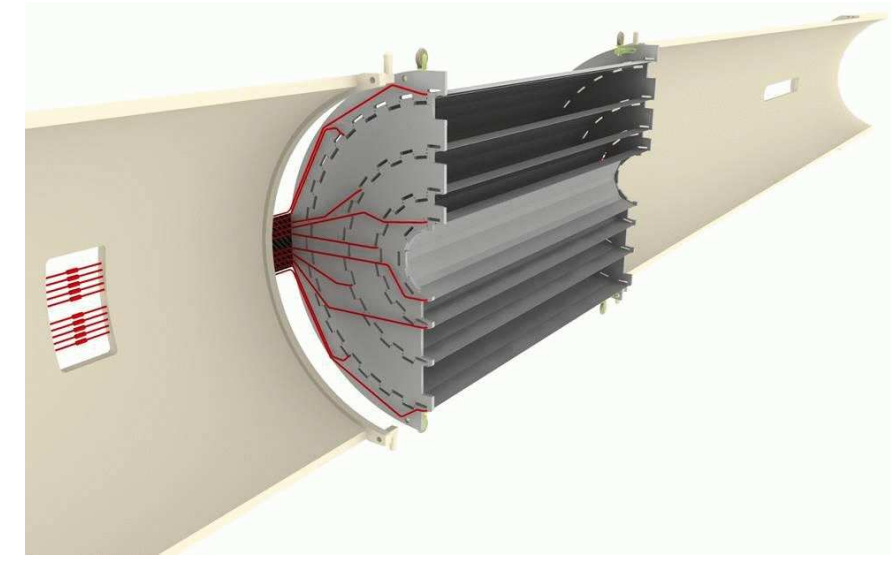

Figure 3: Drawing of one half of the upgraded pixel barrel system. The two half supply tubes can be seen with the 4 layer pixel half barrel in the middle, connected with a carbon fi ber hinge that allows for the desired bending during the installation process. The small cooling lines for the barrel detector are shown.

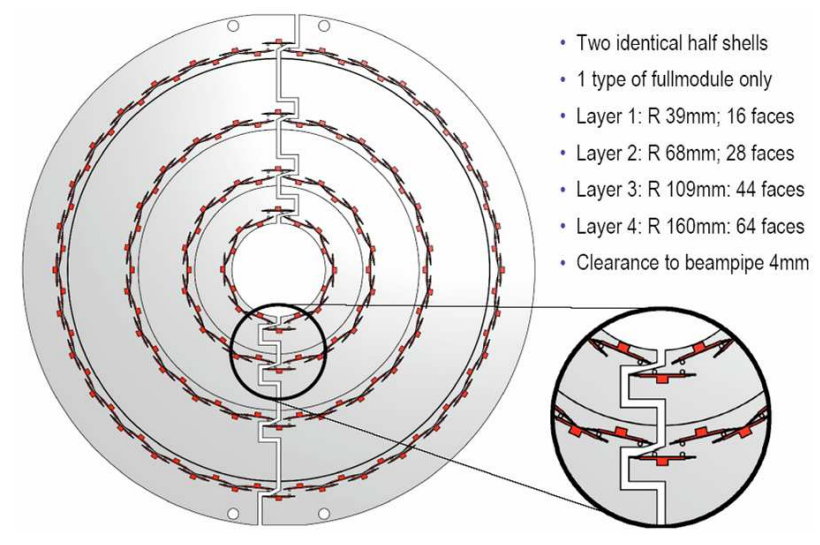

Figure 4: Cross sectional view perpendicular to the beam axis of the upgraded pixel barrel system. Both halves are shown.

The mechanics is made of 200 micron thick carbon fiber sheets glued onto stainless steel tubes carrying the cooling fluid. At both ends there are two end flanges made from a sandwich of carbon 
fiber and Airex foam. With the use of $\mathrm{CO}_{2}$ as coolant a very lightweight and effective cooling system can be built. Each detector half shell has 10 cooling loops in total of which one half is routed through each of the two connected supply tubes. Each loop serves 36 to 40 modules and has a total unfolded length of about $5 \mathrm{~m}$. The loops are assembled from $530 \mathrm{~mm}$ long straight sections alternating with pre-bended 180 degrees sections soldered together. The stainless steel tubing has $1.5 \mathrm{~mm}$ inner diameter with a wall thickness of $50 \mu \mathrm{m}$ on the straight sections and $1.6 \mathrm{~mm}$ inner diameter with $100 \mu \mathrm{m}$ wall thickness at the bent sections. Pressure tests on several prototypes up to 100 bar showed no problems. The structure is designed for a quick replacement of the innermost layers after the modules have reached the end of their lifetime due to radiation damage.

\subsection{Forward Mechanics}

The forward part of the upgraded pixel detector consists of six half disks at each side of the barrel part, twelve in total. Figure 5 shows a drawing of a half disk. The modules on each half disk are arranged in two half rings, with 44 modules in the inner and 68 modules in the outer ring. The modules are mounted on a so called blade made of carbon fiber enclosed pyrolytic graphite. To ensure an overlap in phi, one module is located on each side of a blade. The blades themselves are supported by two carbon fiber half rings in which the cooling loops are embedded. The services of the endcap detector are supported by four supply half tubes similar to the barrel part.

\section{Upgraded Pixel Module Design}

The upgraded pixel detector uses only one module design for both, the barrel and the forward detector. This makes the production and integration easier and simplifies the testing of the modules and the integrated structures. The module is based on the design of the barrel module in the existing detector as described in [4]. The optimized design foresees a couple of changes on almost every component of the module (see fig. 6):

- Removal of silicon nitride base strips: This not only reduces the material budget of the modules by $14 \%$ but also allows for a better thermal contact of the module to the structure and gives smaller temperature difference between the coolant and the sensor. Without the base strips as support the modules will be clamped onto the structure with a layer of thermal grease in between to avoid stress at the delicate bump bonding interface between the readout chips and the sensor.

- Sensor made from $285 \mu \mathrm{m}$ thick DOFZ-silicon: The limited radiation tolerance makes a regular replacement of the innermost layer necessary. In the TDR [6] the present sensors have been specified to a maximum fluence of $6 \times 10^{14}$ pions $/ \mathrm{cm}^{2}$ corresponding to an integrated luminosity of $\approx 1000 \mathrm{fb}^{-1}$ or roughly one year of LHC operation at a peak luminosity of $2 \times 10^{34} \mathrm{~cm}^{-2} \mathrm{~s}^{-1}$. Those numbers are very conservative. Recent measurements [7] on full modules indicate that the modules are suitable for NIEL doses above $10^{15} \mathrm{n}_{\mathrm{eq}}$. Further studies are ongoing. Also, the decision on the exact sensor material can be deferred up to the start of the production. We expect to replace the innermost layer every 2-3 years. 


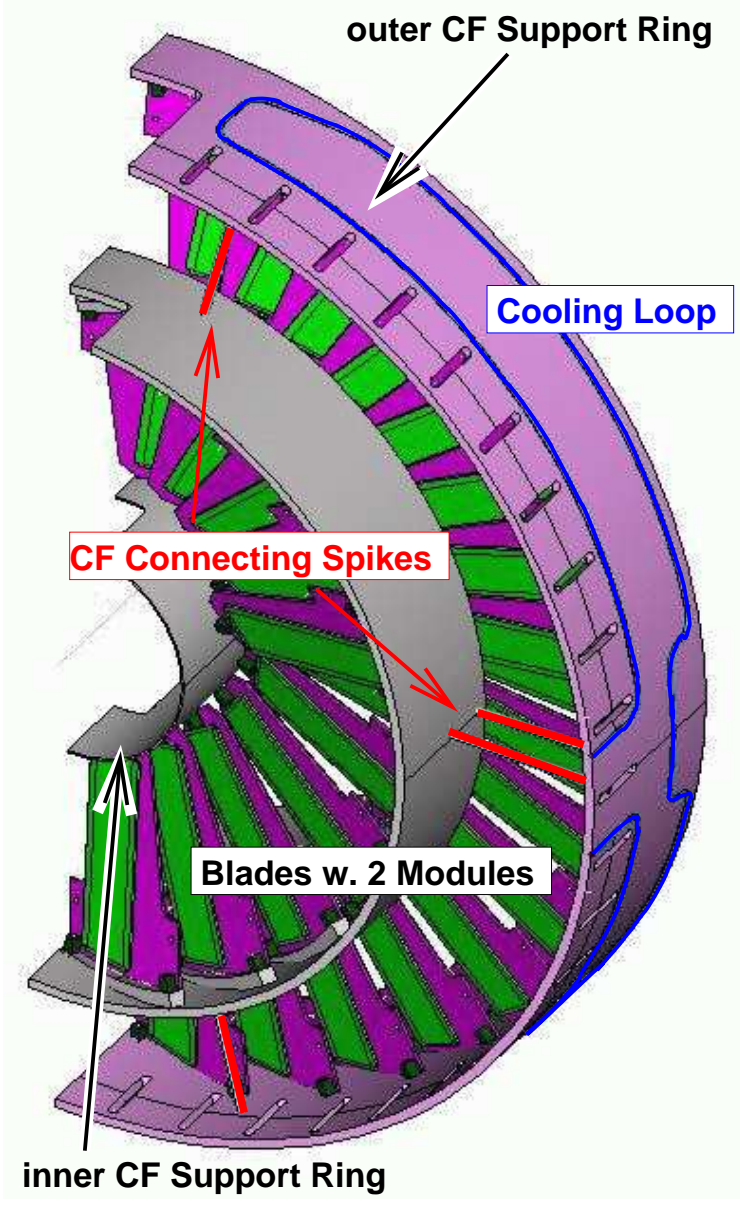

Figure 5: Drawing shows a forward pixel half disks. The two rings of blades that support one detector module on each side can be seen together with the half cylinders that support those blades and carry the cooling loops.

- 16 readout chips (ROCs) with 52x80 pixels of size $150 \times 100 \mu \mathrm{m}^{2}$ : Thinning the readout chips to $75 \mu \mathrm{m}$ leads to a gain of $11 \%$ in material budged for the price of a more demanding handling of the chips and assembled modules.

- High Density Interconnect (HDI), a flexible low mass 3 layer PCB (flex print): Another $18 \%$ in material budget can be saved by redesigning the HDI using SMD components of the smallest size together with the abandonment of the HV filter capacitor used in the current design ${ }^{1}$. It also greatly reduces the vulnerability of modules to beam incidents where a large deposition of charge in the sensor can lead to high voltages appearing at the amplifier inputs.

- Token Bit Manager chip (TBM) controlling the readout of the ROCs: a new purely digital version of the control chip will be used, capable of $320 \mathrm{MHz}$ transmission of the readout data [8].

\footnotetext{
${ }^{1}$ Measurements at the present system have shown, that the impedance on the HV supply is large enough to decouple noise effectively
} 


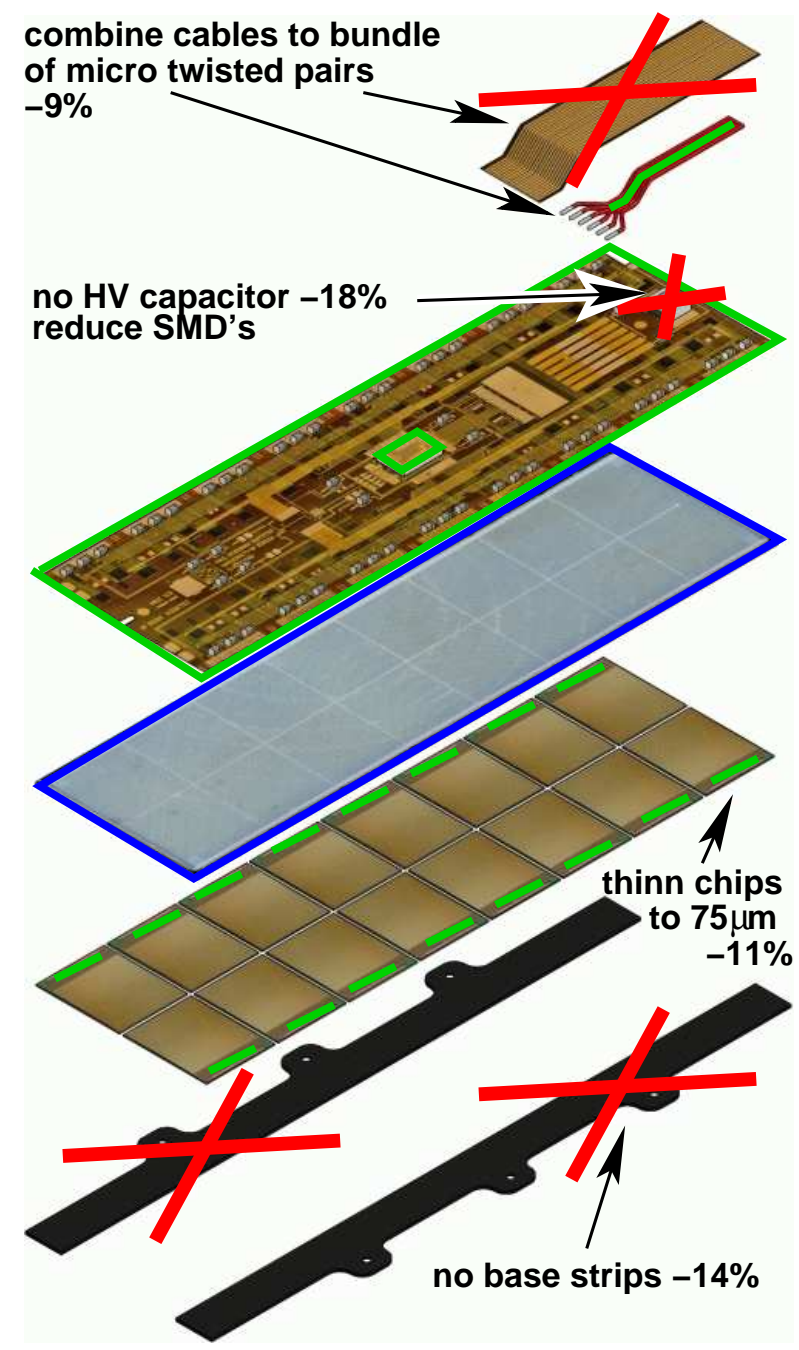

Figure 6: sketch of pixel barrel module showing components being removed or changed. Numbers are savings in material budget with respect to the existing module design.

- Combined signal- and power-cables in form of a bundle of micro-twisted-pair cables: For signals and power distribution low mass copper cladded aluminum wires will be used. The signals are transferred via 12 wires of $125 \mu$ m outer diameter. The 6 power lines have twice the diameter. Both together create $44 \%$ less material effect than the current cables. All wires are bundled together. The total length will be about $1 \mathrm{~m}$ which allows moving the port cards and optohybrids to the edge of the active volume of the detector.

All changes together will reduce the material budget of a module by $44 \%$ plus an additional reduction in the spikes of the material distribution around $|\eta|=1.6$ and 2.0 because the connectors and portcards are moved out of the active volume (see fig. 7). 


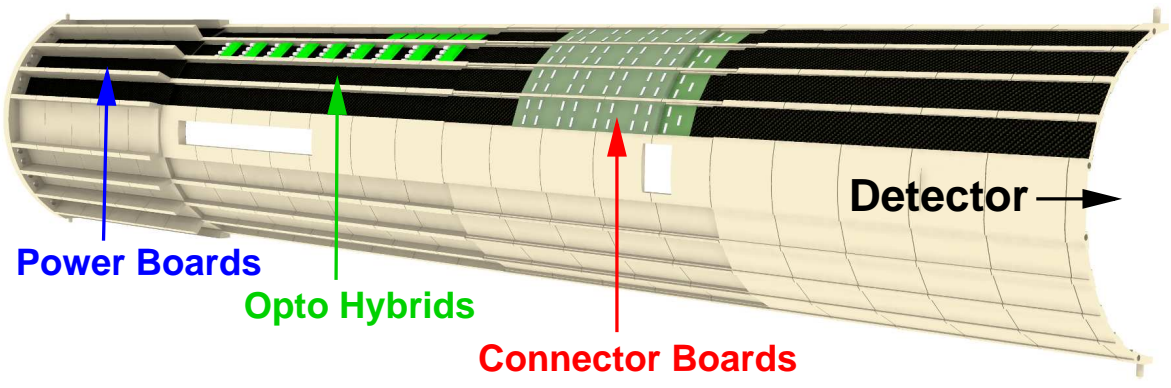

Figure 7: Drawing showing the upgraded barrel supply tube. The optical transceivers together with the power boards are moved out towards higher eta regions. Only the patch panel accepting the module cable plugs is partly located in the detector acceptance.

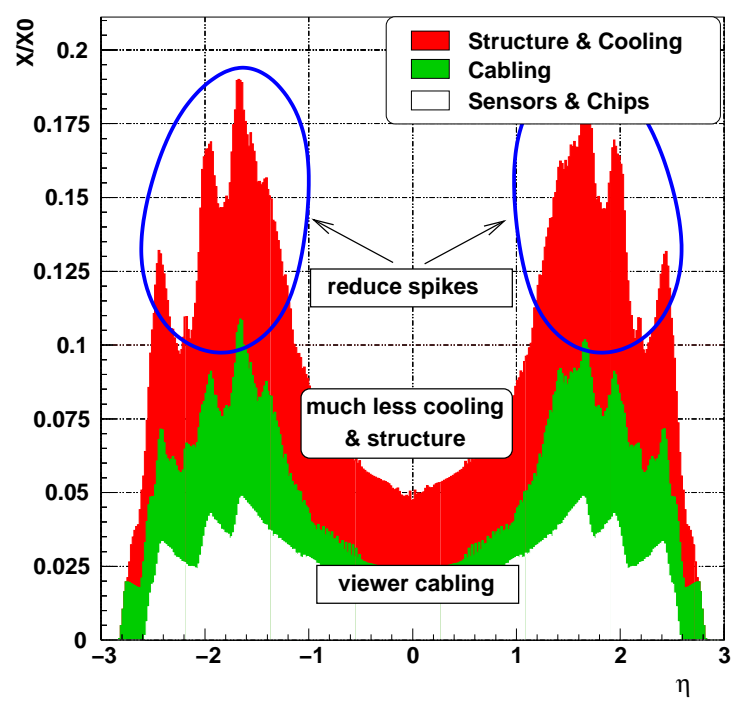

Figure 8: Material budget of the current pixel barrel system with indicated savings

\section{Material Budget}

Figure 8 shows the material distribution of the current pixel detector including support structure, modules, cables and electronics. There are noticeable spikes around $|\eta|=1.6$ due to connector boards on the detector endflange and opto hybrids on the supply tube. This will be largely reduced by connecting modules directly to the supply tube and moving all electronics components away from the interaction region.

The biggest reduction in material budget is achieved through the change in the cooling system with a lower mass coolant and the finer pipes. Figure 9 shows the material budget distribution for the innermost layer of the support structure including the coolant. With $0.27 \%$ radiation length the new structure has only $1 / 3$ rd of the material budget from the currently mechanics.

Figure 10 shows the different contributions to the material budget of the present module. In 
Material budget of layer 1 barrel mechanics

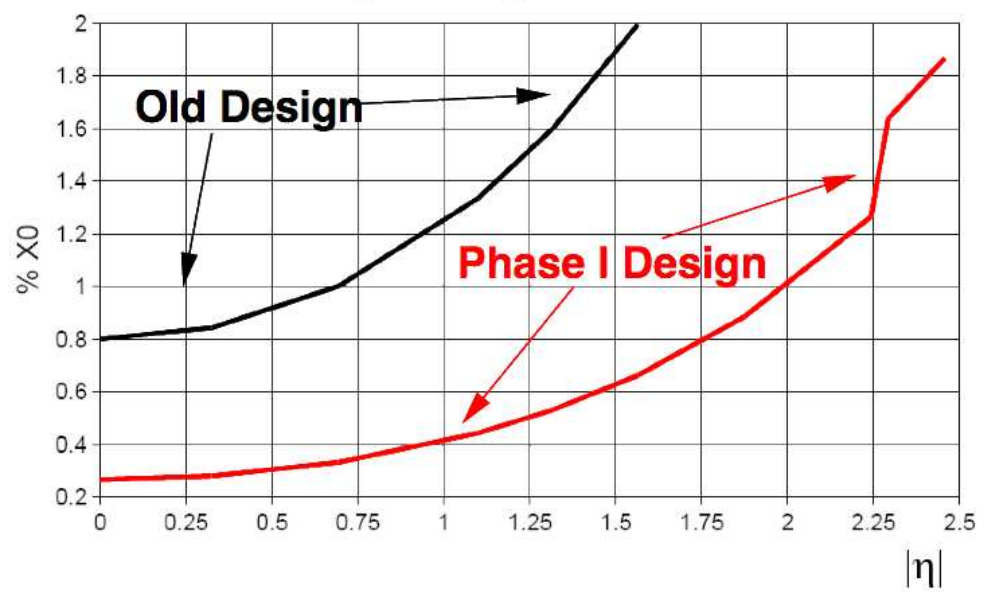

Figure 9: Comparison of the material budget distributions for the support structure of the innermost barrel layer including the coolants. With $0.27 \%$ radiation length at $\eta=0$ the new mechanics has only about $34 \%$ of the material budget of the present structure.

total this is $1.25 \%$ radiation length. In the new module design this will be reduced by $44 \%$. The combined reduction per layer (structure and electronics) will amount to about $50 \%$ in the barrel part with additional savings in the spikes of the current material budged distribution, where the endring prints, module plugs and cooling manyfolds of the current detector are located. The forward pixel detector is aiming for similar savings. The design is still under development.

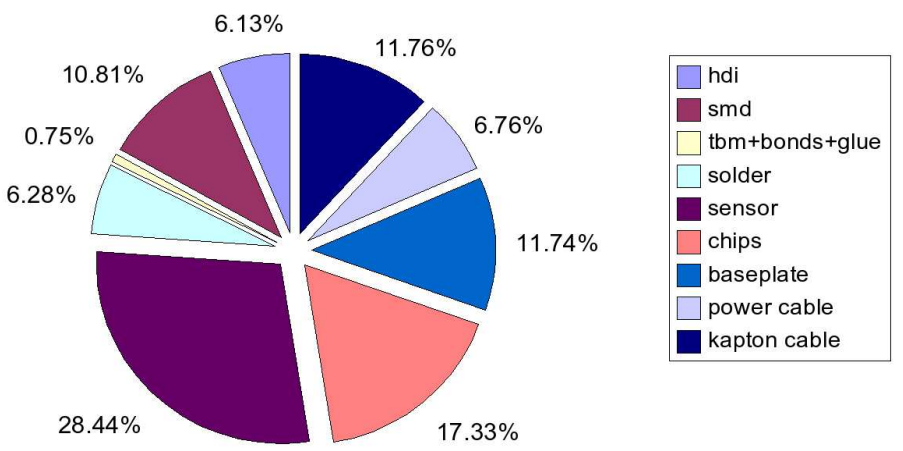

\section{total $1.25 \% \mathrm{X} / \mathrm{X}_{0}$ per Module}

Figure 10: Distribution of the material budget of one detector module. The whole module contributes $1.25 \%$ radiation length to the material budget.

\section{Readout}

The data rate per layer will roughly be doubled after the year 2018. Table 1 summarizes the expected data rates at a peak luminosity of $2 \times 10^{34} \mathrm{~cm}^{-2} \mathrm{~s}^{-1}$ for the 4 barrel layers. The data 
transmission from the module to the counting room as well as the communication inside a module will therefore be changed from a $40 \mathrm{MHz}$ analog to a $320 \mathrm{MHz}$ digital protocol. The transmission inside a module is organized in the following way: The two input channels of the TBM accept the serial digital readout of 8 ROCs each. The readout speed will be $160 \mathrm{MHz}$. Those two channels are then multiplexed on the output channel of the TBM which runs at $320 \mathrm{MHz}$. The TBM drives the $1 \mathrm{~m}$ long twisted pair output line up to the laser driver boards, where the electrical transmission is converted in an optical one. One electrical output of the TBM corresponds to one optical output fiber.

More information about the digital link and the transmission protocol can be found in [8].

Table 1: Estimated data rates and number of optical links for the 4 barrel layers at an instantaneous luminosity of $2 \times 10^{34} \mathrm{~cm}^{-2} \mathrm{~s}^{-1}$ and a fi rst level trigger rate of $100 \mathrm{kHz}$.

\begin{tabular}{|l|c|c|c|c|}
\hline Layer & 1 & 2 & 3 & 4 \\
\hline Pixel fluence $\left[\mathrm{MHz} / \mathrm{cm}^{2}\right]$ & 224 & 96 & 48 & 27 \\
\hline Hits per trigger per module & 68 & 31 & 15 & 8.4 \\
\hline Number of links per layer & 128 & 224 & 352 & 512 \\
\hline MBit/s per link & 194 & 93.6 & 55.9 & 40.0 \\
\hline
\end{tabular}

\subsection{ROC}

With the higher particle rate the inefficiency of the present ROC would be unacceptably high. Therefore, modifications to the chip periphery will be made, mainly enlarging the existing buffer arrays and the introduction of a FIFO buffer for L1 trigger verified hits waiting to be read out. Detailed data flow simulations have been made to estimate the ROC inefficiency at different luminosities. Starting from reconstructed GEANT4 hits ${ }^{2}$, the data flows inside the ROC has been simulated in the time domain. The ROC model in the software closely follows the real hardware architecture. Table 2 shows the expected inefficiencies averaged over an LHC fill assuming a peak luminosity of $2 \times 10^{34} \mathrm{~cm}^{-2} \mathrm{~s}^{-1}$, a beam lifetime of $10 \mathrm{~h}$ and an LHC turnaround time of $5 \mathrm{~h}$.

Together with the introduction of the digital data transmission inside the module the following changes to the ROC will be made:

- include ADC to digitize the pulse height

- add a PLL generating a $160 \mathrm{MHz}$ clock out of the $40 \mathrm{MHz}$ clock that is provided to the chip.

- increase the timestamp and data buffers considerably

- introduce an additional readout buffer

More information about the changes in the ROC design can be found in [8].

\footnotetext{
${ }^{2} \mathrm{~A}$ mix of minimum bias events together with top and $\mathrm{W}$ production and high $\mathrm{p}_{\mathrm{T}} \mathrm{QCD}$ jets have been used
} 
Table 2: Estimated ineffi ciencies for the 4 barrel layers averaged over a LHC fil 11 assuming an instantaneous luminosity of $2 \times 10^{34} \mathrm{~cm}^{-2} \mathrm{~s}^{-1}$, a luminosity lifetime of 10 hours and a fi rst level trigger rate of $100 \mathrm{kHz}$.

\begin{tabular}{|l|c|c|c|c|}
\hline Layer & 1 & 2 & 3 & 4 \\
\hline Inefficiency [\%] & 2.1 & 0.38 & $\approx 0.2$ & $<0.1$ \\
\hline
\end{tabular}

\section{Powering}

In the existing system the power demands of the ROCs is $210 \mathrm{~mW} / \mathrm{cm}^{2}$. For the 11520 ROCs in the barrel this corresponds to a total of $1.6 \mathrm{~kW}$. Due to cable losses of $1.5 \mathrm{~kW}$ the total rack power today is $3.2 \mathrm{~kW}$. The upgraded pixel detector will have about $70 \%$ more ROCs. The power density at a higher luminosity of $2 \times 10^{34} \mathrm{~cm}^{-2} \mathrm{~s}^{-1}$ will be $280 \mathrm{~mW} / \mathrm{cm}^{2}$. This makes a total front end power for the barrel of $3.6 \mathrm{~kW}$. While the services for the third endcap disks have been foreseen from the beginning, the power for the 4 layer barrel detector must be supplied via the existing services designed for 3 layers only. In order to keep the present cables and power supplies two measures have to be taken:

- To reduce the cable losses the use of DC to DC converters with a voltage conversion rate of 2:1 is foreseen. The converters will be located on the far end of the supply tubes, far away from the detector acceptance. With those converters installed, the cable losses between the power supply and the supply tubes can be reduced from $4.5 \mathrm{~kW}$ without converters to $1.6 \mathrm{~kW}$ at a luminosity of $2 \times 10^{34} \mathrm{~cm}^{-2} \mathrm{~s}^{-1}$ which is similar to the losses today. With a conversion efficiency of $80 \%$, the total rack power is increased by about $90 \%$ to $6.1 \mathrm{~kW}$.

- In order to supply enough power to the detector even at the higher luminosities envisaged in the upgrade scenarios, the firmware of the power supplies has to be modified. With this modification it is possible to set higher voltages and currents than needed for the actual detector. The power output of the present power supplies is sufficient for the new system.

With the two modifications it is possible to run the upgraded pixel detector with the existing power supplies and cables currently used up to a luminosity of $3-4 \times 10^{34} \mathrm{~cm}^{-2} \mathrm{~s}^{-1}$. This includes sufficient reserve for special running phases like the start-up of the detector.

\section{Summary}

The expected luminosity buildup in the years up to 2018 makes a replacement of the pixel system necessary. The whole module readout chain will be modified to better suit the higher occupancy environment of the upgrade scenario. This includes the introduction of a $320 \mathrm{MHz}$ digital data transmission via small diameter twisted pair cables. The consequent reduction of mass in the module design and the use of a $\mathrm{CO}_{2}$ cooling system makes it possible to design a four layer pixel upgrade system with about $50 \%$ less material budget per layer with respect to the existing one. To ensure the powering of the additional modules with the existing power supplies, a stage of DC to DC converters is foreseen at the very end of the supply tubes. 


\section{References}

[1] CMS Collaboration, The CMS experiment at the CERN LHC, 2008 JINST 3 S08004, chapter 3

[2] LHC Performance Workshop - Chamonix 2009

[3] CMS Collaboration, CMS Expression of Interest in the SLHC, CERN/LHCC 2007-014

[4] S. König et al., Building CMS pixel barrel detector modules, Nucl. Instrum. Meth. A582 (2007) 776-780

[5] H.C. Kästli, A low mass pixel detector upgrade for CMS, 2010, accepted for publication in Proceedings of Science

[6] CMS Collaboration, The Tracker Project. Technical Design Report, 1998, CERN/LHCC 98-6

[7] T. Rohe et al., Radiation hardness of CMS pixel barrel modules, Nucl. Instrum. Meth. A (2010), in press. Available online at www.sciencedirect.com.

[8] Beat Meier et al., Pixel Front-end Development for CMS, these proceedings 